Afrique SCIENCE 05(1)(2009) 99 - 113

ISSN 1813-548X

\title{
Modélisation de la pollution par les nitrates de la nappe de Berrechid, au Maroc
}

\section{Karima EL BOUQDAOUI ${ }^{\prime *}$, Mostafa AACHIB ${ }^{2}$, Mohammed BLAGHEN' et Sanaa KHOLTEI ${ }^{3}$}

\author{
'Université Hassan II, Faculté des Sciences, Département biologie, Ain Chock, \\ B.P. 5366, Maârif, Casablanca, Maroc \\ ${ }^{2}$ Ecole Hassania des Travaux Publics, B.P. 8108, Casablanca-Oasis, Maroc \\ ${ }^{3}$ Université Hassan ${ }^{\text {er }}$, Faculté des Sciences et Techniques, Settat, Maroc
}

*Correspondance,courriel:karimaelbouqdaoui@hotmail.com

\section{Résumé}

La nappe de Berrechid a toujours suscité un intérêt particulier en tant qu'une source principale d'irrigation et d'alimentation en eau potable dans la région. Cependant, de nombreux indices indiquent une dégradation de la qualité de son eau, contaminée, notamment par les nitrates dont la concentration est excessive. L'analyse de l'eau a montré que, durant l'année 2004, les teneurs en nitrates ont dépassé la valeur admissible $(50 \mathrm{mg} / \mathrm{L})$ dans $70 \%$ de l'ensemble des puits de contrôle. Ces concentrations élevées mettent en évidence un lessivage d'importantes quantités d'engrais azotés vers les eaux souterraines, auxquelles s'ajoutent la présence d'une décharge non contrôlée et les eaux usées d'origine domestique et industrielle.

L'évolution des teneurs en nitrates dans la nappe a été simulée sur une période de 22 ans, de 1993 à 2015, à l'aide des codes numériques MODFLOW et MT3DMS, intégrés dans le logiciel GMS. Les résultats de la modélisation numérique montrent une dégradation continue et globale de la qualité de la nappe par les nitrates, sauf dans la partie sud-est à proximité de la frontière de réalimentation de l'aquifère par le plateau de Settat.

Mots-clés : Modélisation numérique, GMS, MODFLOW, MT3DMS, pollution, nitrates, eav souterraine, Berrechid 


\section{Abstract}

\section{Modeling of nitrate pollution of Berrechid's groundwater (morocco)}

The Berrechid's groundwater is of a high interest because it is a major source of drinking water and irrigation in the area. However, several factors indicate that the quality of its water is very poor, most notably due to high concentrations of nitrates. Water analysis showed that, during the year 2004, nitrates exceeded the acceptable concentration of $50 \mathrm{mg} / \mathrm{L}$ in $70 \%$ of the controlled wells. Such high concentrations of nitrates suggest an important leaching of substantial quantities of nitrogen-rich products into the subsoil water. In addition, the presence of uncontrolled landfill and other polluted rejections in the surrounding area is likely to contribute to water pollution as well. In the present study, using a numerical code MT3DMS, integrated in GMS software, we simulated the evolution of nitrates content in the groundwater over a period of seventeen years ranging from 1993 to 2015. The numerical modeling data show a continuous and general decrease of water quality over the whole area, except in the south-eastern zone juxtaposing the recharge boundary of the aquifer.

\section{Keywords : Numerical modeling, GMS, MODFLOW, MT3DMS, pollution, nitrates, groundwater, Berrechid.}

\section{Introduction}

La nappe de Berrechid est située au sud de la ville de Casablanca. Elle se distingue des autres nappes de la région par l'importance de son étendue qui avoisine $1500 \mathrm{~km}^{2}$. Elle s'insère dans le quadrilatère formé par les villes de Settat, d'El Gara, de Mediouna et le centre de Bouskoura (Figure ク).

Cette nappe a donc fait l'objet de plusieurs études quantitatives [1-3]. Les activités agricoles et industrielles, de plus en plus intensives, auxquelles s'ajoutent une croissance démographique importante et une pluviométrie déficitaire, ont contribué à la diminution des réserves en eau souterraine de cet aquifère et à la dégradation de leur qualité. Parmi les agents chimiques susceptibles d'être à l'origine de la pollution de la nappe phréatique de la plaine de Berrechid figurent les nitrates, divers sels ef les métaux lourds [4].

Le but de ce travail est d'apporter une contribution à la modélisation de l'aquifère de Berrechid afin de délimiter l'étendue de la pollution de la nappe par les nitrates. 


\section{Matériel et méthodes}

\section{2-1. Domaine d'étude}

\section{2-1-1. Cadre géologique, climatologique et hydrologique}

Le climat est directement influencé par la proximité de l'océan. La pluviométrie moyenne annuelle s'élève à $373 \mathrm{~mm}$, avec une période pluvieuse d'octobre à mars. La température présente de forts écarts entre l'hiver et l'été avec une moyenne des maxima de $24,9^{\circ} \mathrm{C}$ et des minima de $9,6^{\circ} \mathrm{C}[7]$.

Du fait de la configuration structurale et topographique de la plaine, le réseau hydrographique est endoréique ; il est constitué, du Nord-Est au Sud-Ouest, par les oueds Asseïla, Aïda, El Ahmeur, Mazere,Tamdrost et Boumoussa (Figure I).

La plaine de Berrechid se présente en surface comme une fosse de subsidence, limitée au sud par les calcaires marneux du Crétacé, et ailleurs par des formations primaires constituées de schistes et de quartzites. Les terrains de remplissage sont formés de grès marins et dunaires du Pliocène [5] (Figure 2).

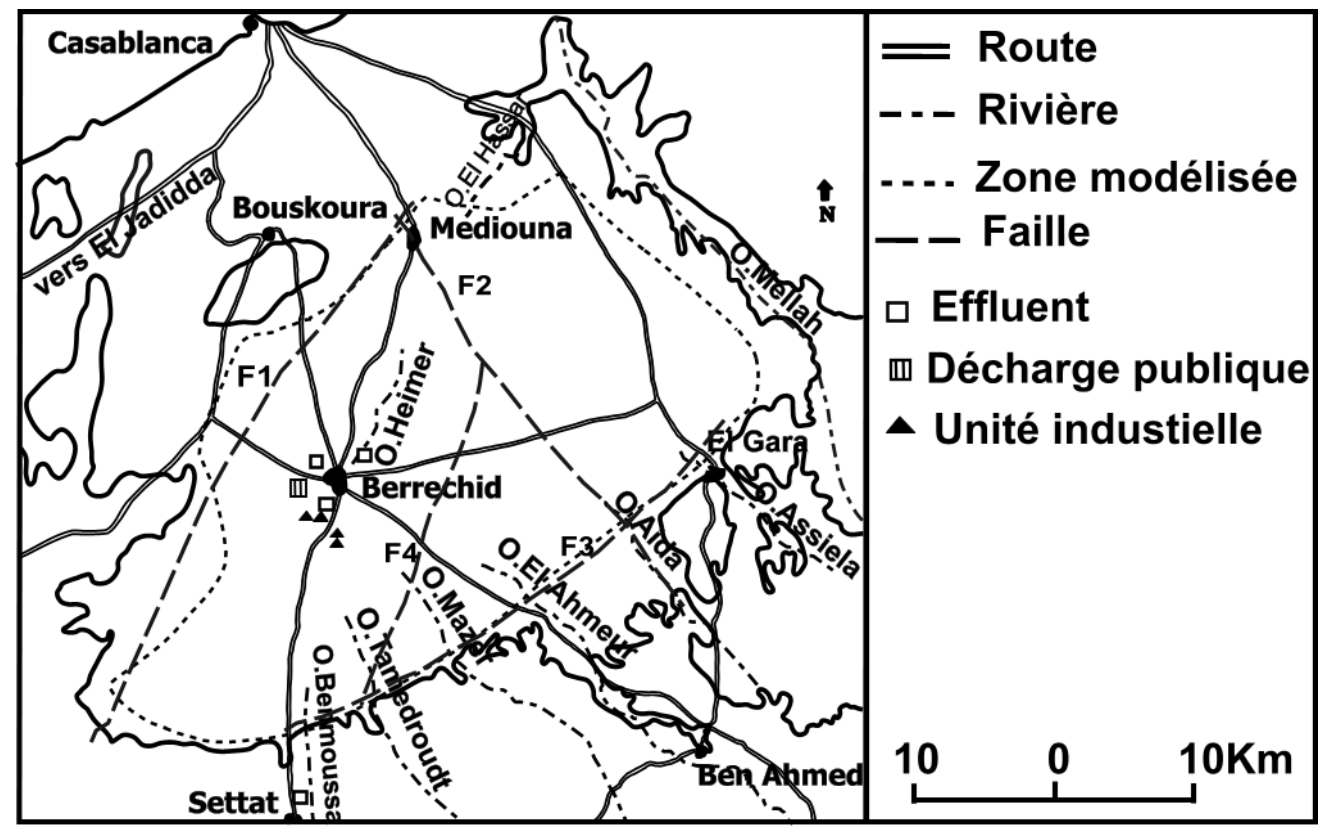

Figure 1 : Carte de localisation [I, 4]. 


\section{2-1-2. Qualité des eaux souterraines}

La qualité de l'eau de la nappe de Berrechid a toujours suscité un intérêt particulier en raison de son utilisation à la fois pour l'irrigation et pour l'alimentation en eau potable de plusieurs localités. Elle a donc fait l'objet de plusieurs campagnes d'analyses. Nous nous intéressons plus particulièrement aux nitrates car ils font partie des principaux indicateurs de la pollution des eaux souterraines, et aussi en raison de leurs teneurs élevées. Les résultats obtenus entre 1993 et 2004, par I'Agence du Bassin Hydraulique de Bouregreg et de la Chaouia (ABHBC) [8] dans leur réseau de contrôle (Figure 3), par le Ministère Chargé de l'Aménagement du Territoire, de l'Eau et de l'Environnement (MCATEE) [9] et par la Direction Générale de l'hydraulique [1], montrent des valeurs maximales qui varient entre $67 \mathrm{mg} . \mathrm{l}^{-1}$ en 1993 et 175 mg. $\mathrm{l}^{-1}$ en 2004 . Ces derniers dépassent largement la limite maximale admissible de potabilité recommandée par le Maroc [10] et par l'OMS [11], et qui est de $50 \mathrm{mg} / \mathrm{l}$. La présence des nitrates dans les eaux de ces puits peut provenir d'une part, de l'infiltration des eaux usées et d'autre part, du lessivage d'importantes quantités d'engrais azotés et de produits phytosanitaires vers les eaux souterraines. La nature de la couverture (limons argileux rouges) favorise le lessivage des nitrates qui sont également chargés négativement; ceux-ci ne sont donc pas retenus par les colloïdes argileux ou organique du sol, d'où leur grande mobilité.

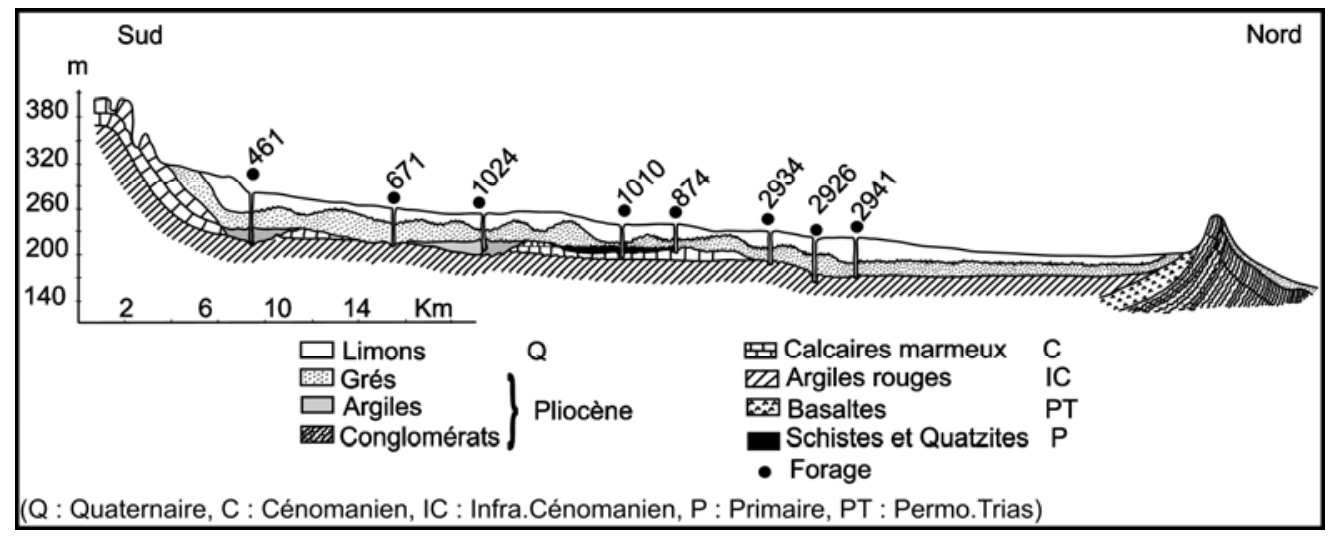

Figure 2 : Coupe géologique sud-nord [6]. 


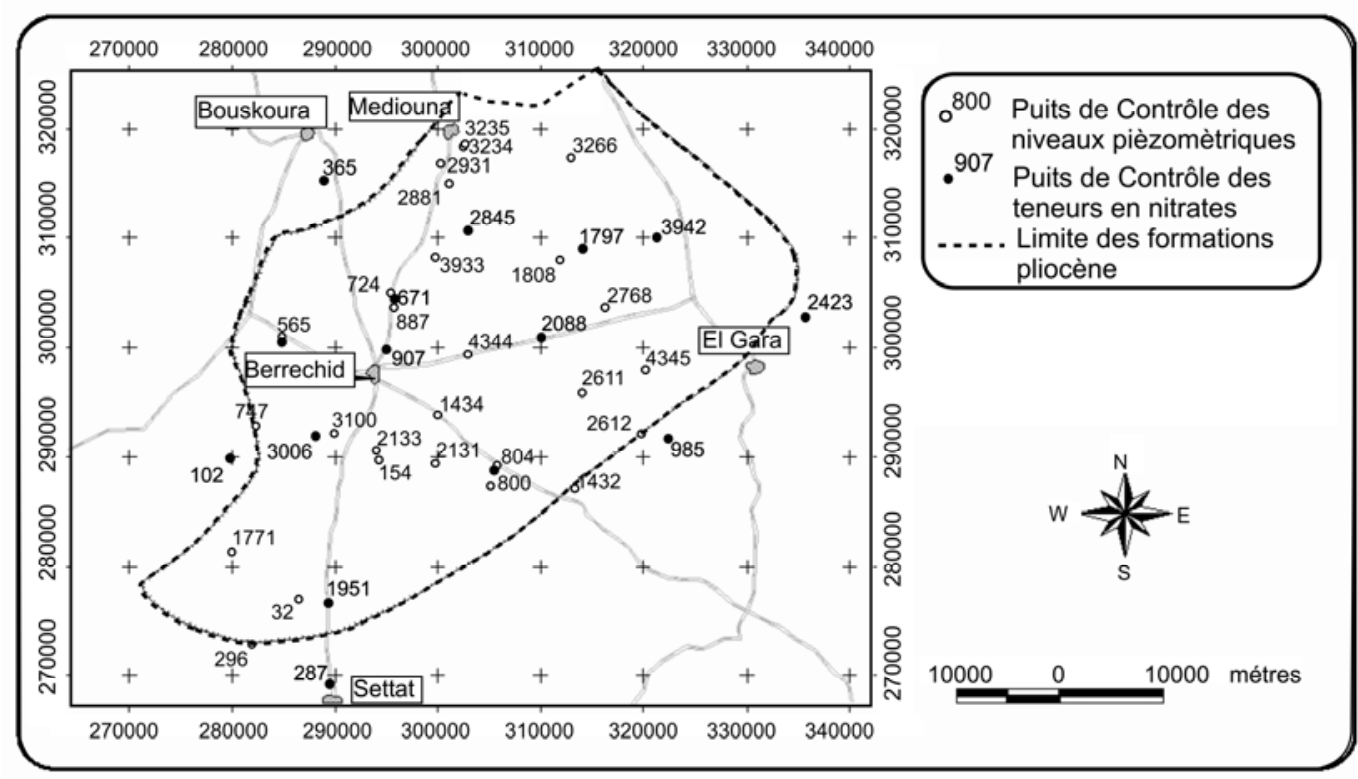

Figure 3 : Réseau des puits de contrôle [8]

\section{2-2. Modélisation de l'écoulement et du transport}

\section{2-2-1. Modèle numérique}

Le modèle numérique a été réalisé en utilisant le logiciel GMS (Groundwater Modelling System) développé par "Environmental Modeling Research Laboratory of Brigham Young University॥ en collaboation avec IU.S. Army Engineer Waterways Experiment Stationll [12]. C'est une interface graphique de différents codes numériques qui permet de traiter divers types de problèmes d'écoulement et de pollution à l'aide des programmes MODFLOW et MT3DMS.

MODFLOW permet de calculer les flux d'eau et les hauteurs piézomètriques à partir de la loi de Darcy et de l'équation de diffusivité. Ces flux sont ensuite utilisés par MT3DMS pour simuler le transport de polluant dans la nappe. Le couplage entre les deux logiciels suppose que les concentrations n'affectent pas les propriétés hydrodynamiques du fluide.

Les transferts d'eau et de polluant miscible en milieu poreux saturé sont décrits par les équations suivantes [13]:

- la loi de Darcy, pour un fluide incompressible : 


$$
V_{i}=-K_{i j} \frac{\partial h}{\partial x_{j}} \quad i, j=1,2
$$

où $V_{i}$ est la vitesse (ou flux) de Darcy et $K_{i j}$ les composantes du tenseur de perméabilité, hétant la charge piézométrique.

La vitesse moyenne réelle (ou vitesse effective) d'écoulement est donnée par $v_{i}=V_{i}$ l $n_{e^{\prime}}$ où $n_{e}$ est la porosité efficace (liée à la réserve en eau mobilisable).

- l'équation d'écoulement (ou de diffusivité):

$\frac{\partial}{\partial x_{i}}\left(T_{i j} \frac{\partial h}{\partial x_{j}}\right)=S \frac{\partial h}{\partial t}+N \quad i, j=1,2$

où $\quad T_{i j}=b K_{i j}$

$T_{i j}$ : composantes du tenseur de transmissivité,

$b$ : épaisseur saturée de l'aquifère,

$S$ : coefficient d'emmagasinement,

$N$ : débit volumique par unité de surface (apports (-) ou prélèvements (+)).

- l'équation de dispersion (ou de transport):

$$
\frac{\partial}{\partial x_{i}}\left(D_{i j} \frac{\partial C}{\partial x_{j}}\right)-\frac{\partial}{\partial x_{i}}\left(v_{i} C\right)-\lambda\left(C+\frac{\rho_{b}}{n_{e}} C_{S}\right)=R \frac{\partial C}{\partial t}-\frac{q}{n_{e}} C^{*}
$$

où $D_{i j}$ sont les composantes du tenseur de dispersion hydrodynamique :

$D_{i j}=\alpha_{T}\|v\| \delta_{i j}+\left(\alpha_{L}-\alpha_{T}\right) \frac{v_{i} \cdot v_{j}}{\|v\|}+D^{*} \quad$ et $\quad\|v\|=\sqrt{v_{i} \cdot v_{i}}$

avec

$C$ : concentration en polluant,

$\alpha_{L, T}$ : dispersivité longitudinale, transversale,

$D^{*}$ : coefficient de diffusion moléculaire en milieu poreux,

$C^{*}$ : concentration en polluant du fluide source entrant ou sortant,

$C_{s}$ : concentration du soluté adsorbé sur la matrice solide,

$q$ : débit volumique par unité de volume (apports (-) ou prélèvements $(+)), q=N / b$,

$\lambda$ : constante de dégradation du polluant,

$R=1+\frac{\rho_{b}}{n_{e}} \frac{\partial C_{s}}{\partial C}$

solide. 
Dans notre cas, la dégradation par dénitrification est supposée nulle $(\lambda=0)$ et l'adsorption négligeable $(R=1)$. La diffusion moléculaire dont la valeur du coefficient est très faible sera également négligée par rapport à la dispersion cinématique.

\section{2-2-2. Paramètres hydrodynamiques et conditions d'écoulement}

Le domaine hydrogéologique modélisé est considéré comme étant un système monocouche, constitué de pores d'interstices. Le choix du modèle conceptuel a été conditionné à la fois par la recherche d'une grande précision, mais aussi par la quantité et la qualité des données disponibles. La préparation des données a été faite à l'aide du logiciel ARCVIEW (outil SIG) selon un maillage régulier de $250 \mathrm{~m} \mathrm{x}$ $250 \mathrm{~m}$. La grille résultante contient 44974 mailles (226 lignes et 199 colonnes). Les conditions aux limites sont de type potentiel imposé (condition de Dirichlet) ou flux nul (condition de Neumann):

- Condition de potentiel imposé d'alimentation au niveau de la limite avec le plateau de Settat au sud et de drainage au nord de la nappe, au nord-ouest et à l'est dans certaines zones le long de la vallée de l'oved Mellah.

- Condition à flux nul à l'ouest au contact des terrains schisteux du primaire et à l'est où les terrains argileux le long de la vallée de l'oued Mellah sont très fortement encaissés.

Pour nos simulations de l'écoulement en régime transitoire, la piézométrie initiale choisie est celle de 1980 qui correspond à la piézométrie obtenue par calage en régime permanent.

La distribution des conductivités hydrauliques résultante de ce calage a mis en évidence trois zones de perméabilité, avec un maximum de $1.710^{-2} \mathrm{~m} / \mathrm{s}$ et un minimum de $3.010^{-5} \mathrm{~m} / \mathrm{s}[14]$ :

- une zones à très faibles perméabilités avec des valeurs inférieures à $10^{-4} \mathrm{~m} / \mathrm{s}$, généralement situées au sud de la plaine. Ce sont les zones les moins transmissives où la nappe circule dans des franges gréseuses peu épaisses.

- une zone à bonnes perméabilités avec des valeurs supérieures à $10^{-3} \mathrm{~m} / \mathrm{s}$. Cette zone est située principalement à l'ouest et au sud ouest de la plaine.

- le reste du domaine correspond à des perméabilités intermédiaires (entre $10^{-4}$ et $10^{\circ}$ $\left.{ }^{3} \mathrm{~m} / \mathrm{s}\right)$.

Pour le calage du modèle hydrodynamique en régime transitoire, une période de 24 ans (de 1980 à 2004) a été choisie en fonction des données piézomètriques disponibles. Les valeurs du coefficient d'emmagasinement issues de ce calage varient entre 5,0 10-4 et 2,0 10.-1 . Trois zones principales ont été distinguées [14] : 
- une zone de faibles coefficients d'emmagasinement située à l'est et dans la partie centrale de l'aquifère avec des valeurs comprises entre $5,010^{-4}$ ef $10^{-3}$. Cette zone correspond en partie aux endroits où la nappe est en charge.

- une autre zone située au nord et au sud avec des valeurs plus élevées, comprises entre $10^{-3}$ et $10^{-2}$.

- le reste de l'aquifère est caractérisé par un coefficient d'emmagasinement compris entre $10^{-2}$ et $2,010^{-1}$.

Ces variations sont dues principalement aux changements latéraux de faciès dans l'aquifère. La porosité efficace est estimée à 3\% [7].

\section{2-2-3. Paramètres dispersifs et conditions de transport}

Pour modéliser le transport des nitrates dans la zone saturée de l'aquifère, des concentrations ont été imposées dans des puits d'injections à partir des mesures annuelles selon les données disponibles dans les puits de contrôle de l'Agence du Bassin Hydraulique de Bouregreg et de la Chaovia (Figure 3). Ailleurs, des concentrations nulles ont été appliquées. L'état initial choisi pour le modèle de transport est celui de 1993.

Les valeurs de la dispersivité longitudinale trouvées dans la littérature, pour un milieu de nature similaire, sont de l'ordre de $100 \mathrm{~m}[15,16]$. Le calage du modèle hydrodispersif a permis de déterminer des valeurs de dispersivité longitudinale $\left(\alpha_{l}\right)$ variant entre $98 \mathrm{~m}$ et $103 \mathrm{~m}$ sur l'ensemble du domaine, le rapport d'anisotropie $\left(\alpha_{\tau} / \alpha_{l}\right)$ étant choisi égal à $0,1[16]$.

\section{Résultats et discussion}

\section{3-1. Evolution du niveau Piézométrique}

L'évolution des niveaux piézométriques de la nappe de Berrechid a été simulée entre 1980 et 2004, dans quelques puits contrôlés par l'Agence du Bassin Hydraulique de Bouregreg et de la Chaouia [8]. On constate un abaissement continu du niveau piézomètrique qui s'intensifie pour atteindre $20 \mathrm{~m}$, surtout au niveau de la zone centrale de l'aquifère (puits 154/28) où se situent les puits d'alimentation en eau potable (AEP), en plus des prélèvements agricoles (figure 4). On note cependant une stabilisation du niveau pour le puits $(800 / 28)$ qui est situé à proximité de la limite de réalimentation au sud de la nappe (figure 4 et 3). (e bilan hydrique déficitaire met en péril les réserves en eau mobilisables de l'aquifère de Berrechid. 
Afrique SCIENCE 05(1)(2009) 99- 113
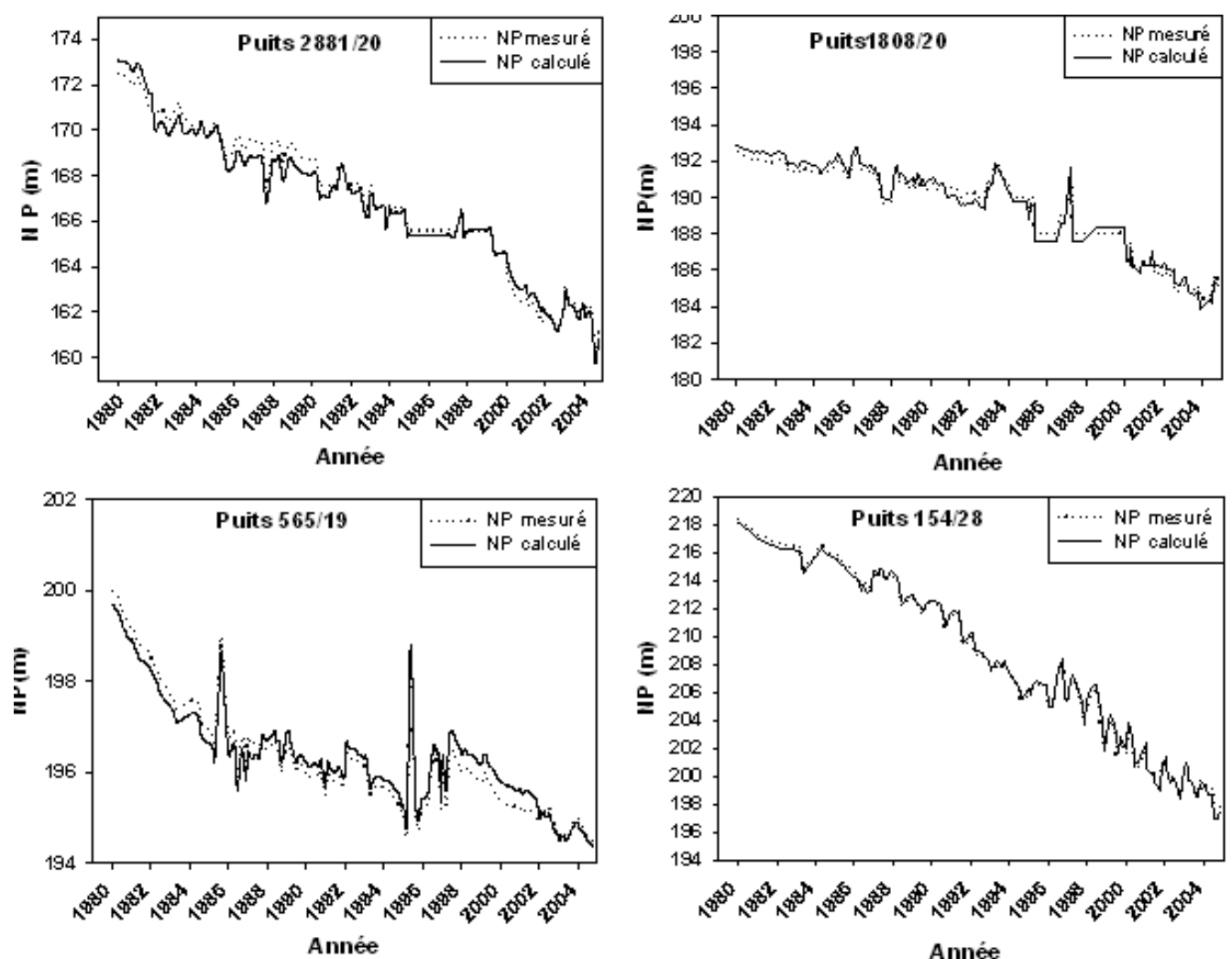

Année
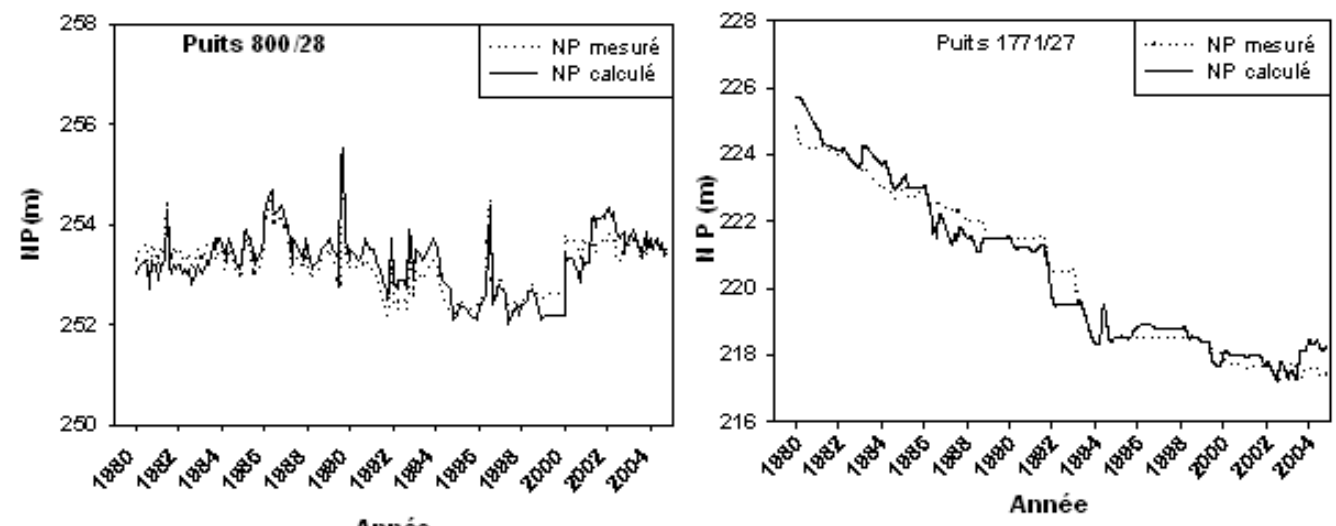

Année

Figure 4 : Comparaison des niveaux piézométriques (NP) mesurés et calculés dans quelques puits du réseau de contrôle [14] 


\section{3-2. Evolution des teneurs en nitrates}

L'évolution des teneurs en nitrates dans la nappe a été simulée sur une période de 1993 à 2004, avec une prédiction en 2015. Les concentrations initiales sont affectées à chaque maille après interpolation des données de la campagne réalisée dans la zone d'étude en 1993 par $A B H B C$ [8]. La modélisation des transferts et le calcul des concentrations sont effectués en $2 \mathrm{D}$; les concentrations sont supposées uniformes sur toute l'épaisseur de la nappe.

Après calage, la comparaison des concentrations mesurées avec celles calculées à l'aide du modèle numérique montre une bonne concordance : les écarts entre ces valeurs demeurent faibles dans la plupart des puits de contrôle $\left(0,02\right.$ à $\left.4 \mathrm{mg} . \mathrm{l}^{-1}\right)$ (Figure 5). On note, entre 2000 et 2002, une augmentation brutale des teneurs en nitrates (Figure 5); ceci est vraisemblablement dô à un apport excessif d'engrais, concomitant à une diminution des précipitations efficaces consécutives à la sécheresse qui a sévi durant cette période.

La qualité globale de la nappe de Berrechid se dégrade de façon notable et continue. Dans la partie Nord-0uest de la ville de Berrechid où se trouvent les puits $671 / 20$ et $2845 / 20$, les concentrations en nitrates s'élèvent de 75 à $175 \mathrm{mg} . \mathrm{L}^{-1}$ (Figure 5). II en est de même au Sud-Ouest de l'aquifère (puits 1951/27) où les concentrations atteignent $125 \mathrm{mg} \mathrm{L}^{-1}$ en 2004 (Figure 6). Ces puits sont situés à proximité de rejets déversés dans les oueds Heimer et Boumoussa qui sont devenus ces dernières années des égouts à ciel ouvert (Figure $ク$ ). L'évolution spatiale montre une diminution notable des teneurs en nitrates au sud-est de la nappe (puits 804/28) (Figure 5, 6 et $\lambda$. Cette partie de la nappe longe la limite d'alimentation au sud du plateau de Settat, à laquelle s'ajoute la recharge à partir d'un certain nombre d'oueds temporaires qui se perdent à l'entrée de la plaine [7].

Les variations spatiales des teneurs en nitrates dépendent de la variabilité des apports liés au contexte environnemental (décharge, effluent, zone agricole ou urbaine, etc). En 1993, les concentrations des nitrates varient entre 10 et $70 \mathrm{mg} . \mathrm{L}^{-1}$; les teneurs maximales ont été observées au nord de la ville de Berrechid où les valeurs varient entre 60 et $70 \mathrm{mg}^{-L^{-1}}$ (Figure 6). En 2004, les nitrates accusent une augmentation nette et importante en cette zone ; les teneurs s'élèvent de $75 \mathrm{mg} \cdot \mathrm{L}^{-1}$ à $175 \mathrm{mg} . \mathrm{L}^{-1}$ (Figure $\pi$. 
Afrique SCIENCE 05(1)(2009)99- 113
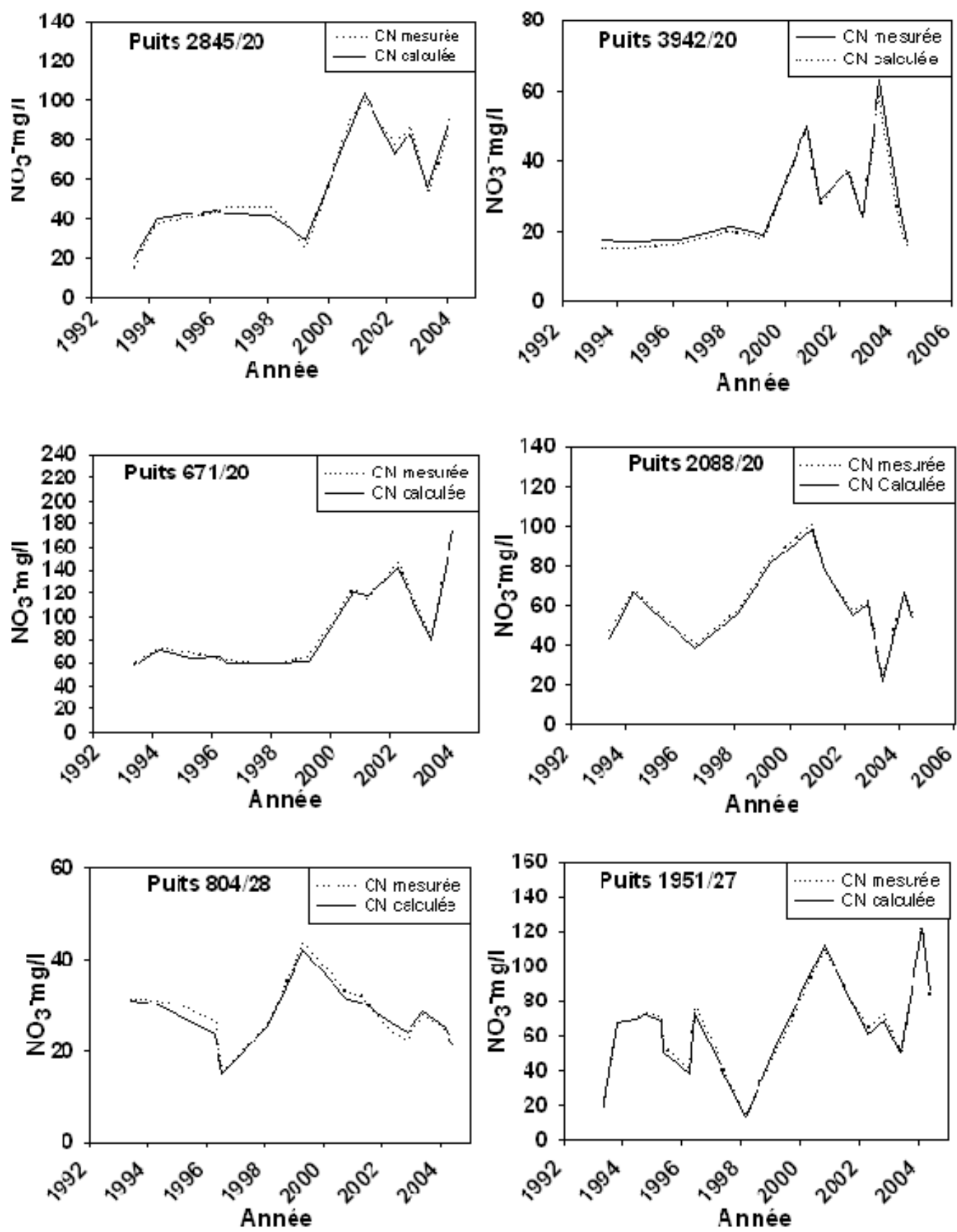

Figure 5 : Simulation de l'évolution des concentrations en nitrates (CN) de 1993 d̀ 2004 des puits de réseau de contrôle. 
Ceci serait probablement dô à un apport par la décharge publique non contrôlée présente dans la région et aux rejets industriels (Figure 1). A l'est et au Sud-Est, les concentrations demeurent en deçà de la valeur limite admissible (i.e, inférieures à $50 \mathrm{mg} / \mathrm{L}$ ) (Figure $\nearrow$.

Par ailleurs, d'autres facteurs semble accélérer l'infiltration des nitrates vers la nappe, notamment l'épaisseur de la couverture qui ne dépasse pas $10 \mathrm{~m}$ au niveau du puits 565/19 à l'ouest et varient entre 10 et $20 \mathrm{~m}$ au niveau des puits $671 / 20$ et 907/20 au centre de la nappe (Figure 3), et la présence de failles (F1, F2 et F4) qui entourent ces zones (Figure П). A l'est, où l'épaisseur de la couverture atteint $50 \mathrm{~m}$, et au sud (à proximité de la frontière de recharge), les concentrations sont en deçà de la valeur limite admissible.

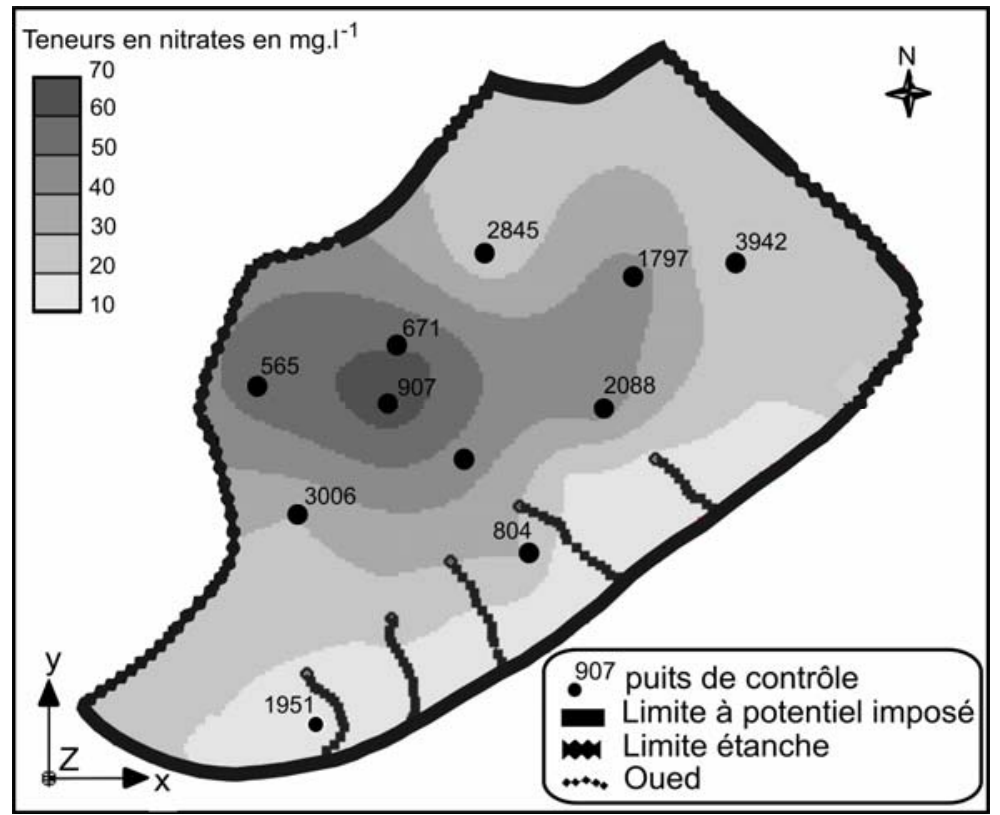

Figure 6 : Répartition des teneurs en nitrates simulées en 1993

La distribution des teneurs en nitrates prédites pour 2015 est réalisée en utilisant des données climatiques (précipitations et températures) entre 2005 et 2015 similaires à celles entre 1994 et 2004. Les résultats obtenus montrent que I 'évolution de la pollution suit la même tendance; elle se manifeste partout dans la nappe sauf au sud-est. Au centre de la plaine et en aval de la zone de Berrechid, la concentration des nitrates dépasse $250 \mathrm{mg}^{-1}{ }^{-1}$ (Figure $\nearrow$ ). 


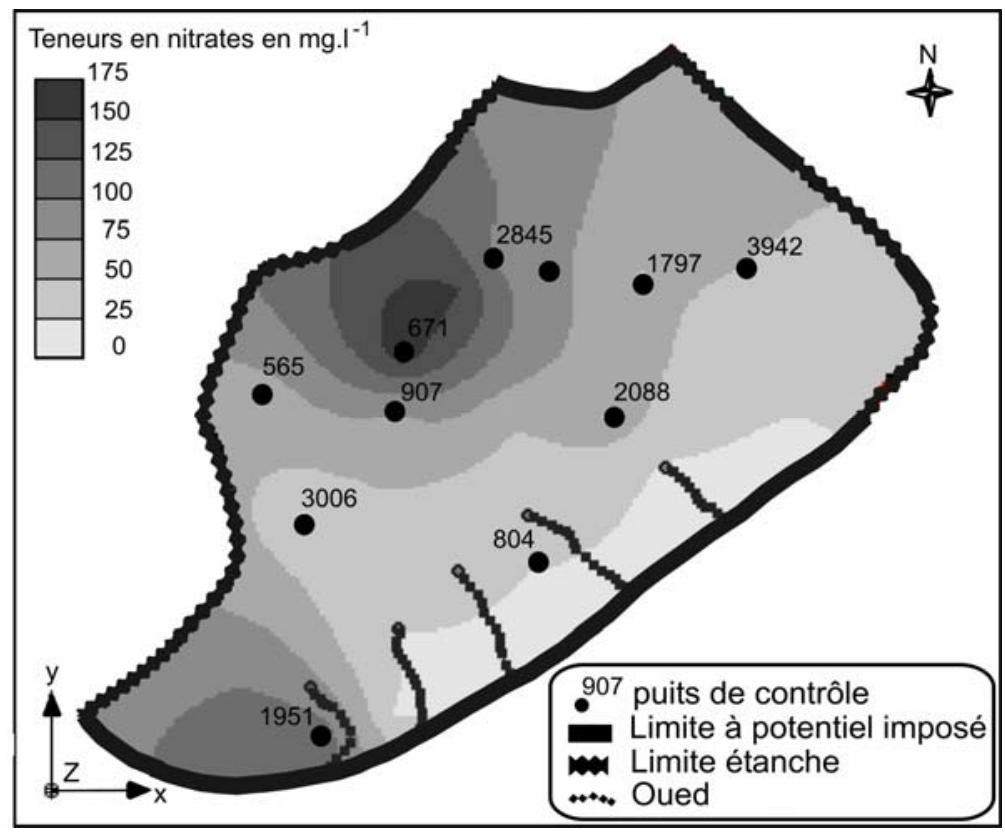

Figure 7 : Répartition des teneurs en nitrates simulées en 2004

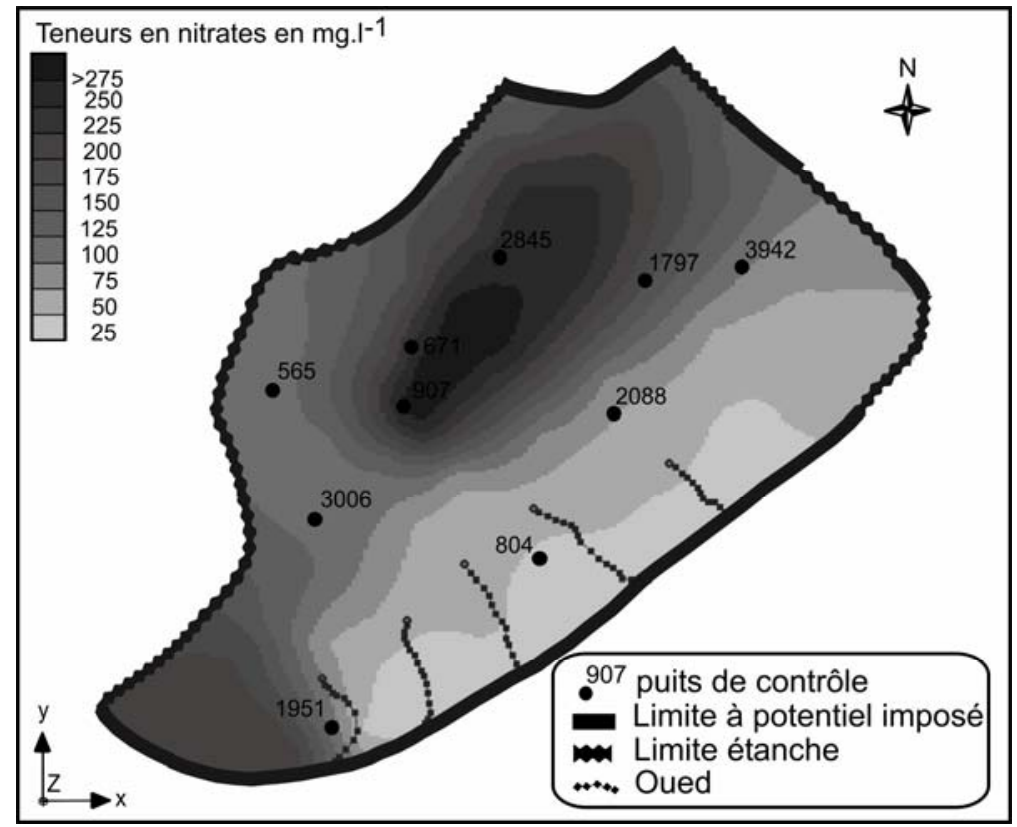

Figure 8 : Répartition des teneurs en nitrates prédites pour 2015 


\section{Conclusion}

La modélisation numérique de l'aquifère de Berrechid nous a permis de délimiter l'étendue de la pollution de la nappe par les nitrates et de mettre en évidence les sites les plus touchés par les pollutions locales.

La nappe d'eau souterraine, dans son ensemble, subit une dégradation de sa qualité au fil des ans depuis 1993, surtout au nord-ouest et au sud-ouest de la plaine. Les résultats de prédiction en 2015 confirment cette tendance, sous les mêmes conditions climatiques ef d'utilisation des sols. Plusieurs phénomènes contribuent à la contamination de cette nappe par les nitrates, à savoir la présence dans la région d'une décharge publique non contrôlée et des rejets d'eaux usées (industrielles et domestiques), en plus de l'utilisation de fertilisants azotés.

La modélisation des phénomènes de transport a aussi mis en évidence la grande sensibilité de la nappe aux apports du plateau de Settat (frontière de recharge au sud de la nappe), en plus de ceux de surface.

\section{Remerciements}

Les auteurs remercient plus particulièrement le Directeur et les Ingénieurs de l'Agence du Bassin Hydraulique de Bouregreg et de la Chaovia, pour leur aide documentaire précieuse et leur disponibilité.

\section{Références}

[1] - Direction Générale de l'hydraulique (DGH), II Carte de sensibilité à la pollution de la région Casablanca-Mohammedia-Settat II, (1994).

[2] - K. El Bouqdaoui, "I Etude géologique et géotechnique de la ville de Settat. Cartographie géotechnique et substances utiles de la province de Settat 11. Doctorat de troisième cycle en géologie, Uni. M ed V. Fac. Sc. Rabat, (1995).

[3] - B. El Mansouri, II Structure et modélisation quantitative de l'aquifère de Berrechid. Validation par l'approche géostatistique II. Doctorat de l'université en géosciences, option : Hydrogéologie quantitative, (1993).

[4] - S. Kholtei, ॥ Plaine de Berrechid ; Caractérisations des eaux usées de Settat et de Berrechid; Evaluation de leurs impact sur la qualité des eaux souterraines et risque toxicologique II. Doctorat d'Etat ès Sciences Physiques. Spécialité : Chimie Appliquée et environnement, Uni. Hassanll. Fac. Sc. Ben M'sik. Casablanca, (2002). 
[5] - R. Hazan and L. Moullard, ॥ Notice hydrogéologique de la plaine de Berrechid II. Office national des irrigations. Services des ressources en eau, Rabat, (1962).

[6] - L. Moullard and R. Hazan, II Nappe phréatique de la nappe de Berrechid II. Assoc. Inter. Hydrol. Sc. Helsinki, Pub. 52, (1960) 105-14

[7] - Direction Générale de l'hydraulique (DGH) (Direction de la recherche et de la planification de l'eau), ॥ Etude de la plaine de Berrechid. Description et analyse II. Mission 1. Edition définitive (Annexes A et B), (1997).

[8] - Agence du Bassin Hydraulique de Bouregreg et de la Chaovia (ABHBC), ISynthèse des études antérieures et actualisation des données hydrogéologiques de la nappe de Berrechid II, (2004).

[9] - Ministère Chargé de l'Aménagement du Territoire, de l'Eau et de l'Environnement (MCATEE), « Etat de la qualité des ressources en eau au Maroc II, (2003).

[10] - Norme marocaine relative à la qualité des eaux d'alimentation humaine. Impression et Edition, Office nationale de l'eau potable (ONEP), (1993)

[11] - 0.M.S. (l'Organisation Mondiale de la Santé), Guidelines for Drinking water quality. 1993.

[12] - Environmental Modeling Research Laboratory, I Groundwater Modeling System GMS Reference Manual II, Birmingham Young University, Birmingham, (1999).

[13] - J. Bear, II Hydraulics of groundwater II, Mc Graw-Hill Inc., (1979).

[14] - G. De Marsily, "Quantitative Hydrogeology II. Academic Press Inc, London, (1996).

[15] - K. El Bouqdaoui, M. Aachib, M. Blaghen and S. Kholtei, "Modélisation de l'écoulement de la nappe de la plaine de Berrechid (Maroc) II. Revue internationale de l'eau "La Hoville Blanche", (Accepté en Novembre 2007).

[16] - S. K. Gupta, K. K. Taji and J.N. Luthin, " A three-dimensional finite element groundwater model. Report UCAL-WRC-C-152. California Water Resour. Cent., Univ. of Califonia, Davis, (1975) 248-925. 